\title{
On Secure Distributed Storage Systems with Exact Repair
}

\author{
Ravi Tandon ${ }^{\dagger}$, SaiDhiraj Amuru, T. Charles Clancy ${ }^{\dagger}$ and R. Michael Buehrer \\ Bradley Department of Electrical and Computer Engineering \\ ${ }^{\dagger}$ Hume Center for National Security and Technology, \\ Virginia Tech, Blacksburg, VA USA \\ Email: $\{$ tandonr, adhiraj, tcc, rbuehrer\}@vt.edu
}

\begin{abstract}
Distributed storage systems (DSS) in the presence of a passive eavesdropper are considered in this paper. A typical DSS is characterized by 3 parameters $(n, k, d)$ where, a file is stored in a distributed manner across $n$ nodes and can be recovered entirely from any $k$ out of $n$ nodes. Whenever a node fails, $d \in[k, n)$ nodes help in repairing the failed node. The focus of this work is on the exact repair capabilities of a DSS, where a failed node is replaced with an identical node. Securing this DSS from passive eavesdropping attacks is studied in this paper. The eavesdropper is capable of wiretapping the repair process of a subset of nodes in the storage system. The main contribution of this paper is the optimal characterization of the secure storage-vs-exact-repair-bandwidth tradeoff region which prior to this work was unknown. We focus on the simplest nontrivial instances of this problem, namely $(n, k, d)=(3,2,2)$ and $(4,3,3)$, and present novel information-theoretic converse proofs that validate these optimal tradeoff regions.
\end{abstract}

\section{INTRODUCTION}

A continuous rise in the volume of data managed across various systems, calls for new storage mechanisms that maintain this data reliably. Distributed storage is the default technique for storing data in all new generation applications. The data from a file is stored in a decentralized manner on several unreliable nodes/disks that when collectively used are capable of recovering the entire file. To ensure robustness to disk failures, the simplest scheme is to replicate and store the data across several disks. For instance, the Google File System (GFS) and the Hadoop Distributed File System (HDFS) store 3 copies of the data across several nodes [1]. While replication is robust to failures, it is not a scalable strategy to store large volumes of data. As an alternative, erasure codes (for instance, Reed-Solomon codes) have been used by Facebook, OceanStore, RAID-6 [2] and others to introduce redundancy into the storage system. However, to repair a failed node, the entire file must be downloaded from the remaining alive nodes. Thus, the repair process for such codes can be excessively bandwidth intensive. To overcome these issues, the concept of regenerating codes for distributed storage systems was introduced by Dimakis et al. [3]. A typical distributed storage system (DSS) consists of $n$ storage nodes each with a storage capacity of $\alpha$ units of data such that the entire file of size $\mathcal{B}$ can be recovered by accessing any $k<n$ nodes. This is called as the reconstruction property of the DSS. Whenever a node fails, $d$ nodes (where $d \in[k, n)$ ) participate in the repair process by sending $\beta$ units of data each. This procedure is termed as the regeneration of a failed node and $\beta$ is referred to as the per-node repair bandwidth.
In [3], by using the concepts of network coding [4], the authors show that the parameters of a DSS must satisfy

$$
\mathcal{B} \leq \sum_{i=0}^{k-1} \min (\alpha,(d-i) \beta) .
$$

Thus, in order to store a file of size $\mathcal{B}$, there exists a fundamental tradeoff between $\alpha$ (storage) and $d \beta$ (total repair bandwidth). However, this tradeoff is in general achievable only for the functional-repair case [3]. In functional repair, a failed node is replaced by a new node such that the resulting DSS has the same reconstruction and regeneration capabilities as before. In particular, the contents of the repaired node may not necessarily be identical to the failed node even though the desirable properties of the DSS are preserved.

In contrast to functional repair, exact repair regeneration requires the repair process to replace a failed node with an identical new node. Exact repair is useful in many practical applications where the data has to be stored intact. The file recovery process is also easier in this case as the reconstruction procedure need not change whenever a failed node is replaced. While characterizing the storage-vs-bandwidth tradeoff for the case of exact repair remains a challenging open problem in general, two extreme points of this tradeoff (depending on whether $\alpha$ or $\beta$ is minimized first) namely, the minimum storage regenerating case (MSR) and the minimum bandwidth regenerating $(\mathrm{MBR})$ case have been studied extensively (see [5], [6] and references therein). Beyond these points, the optimal exact-repair tradeoff for the $(4,3,3)$-DSS was characterized in [7] where it has been shown that the optimal tradeoffs for functional and exact repair do not align.

Securing sensitive data such as personal and financial records from eavesdroppers is necessary to ensure data privacy for the users. Hence a DSS should be secure apart from satisfying the reconstruction and regenerating requirements. Two types of eavesdropping attacks can potentially occur in a DSS, (a) Type-I attack, in which the eavesdropper can wiretap the storage contents of $l$ nodes in the DSS and (b) Type-II attack, in which the eavesdropper wiretaps the contents of the repair data (and thereby the storage content as well) of $l$ nodes in the DSS. Throughout this paper, we assume that $l<k$ since $k$ is the minimum number of nodes required to reconstruct the file of size $\mathcal{B}$. Else, if $l \geq k$, the eavesdropper can recover the file by using the reconstruction property of the DSS.

Cryptographic approaches to ensure data security are based on the assumption that the adversary has limited computational 


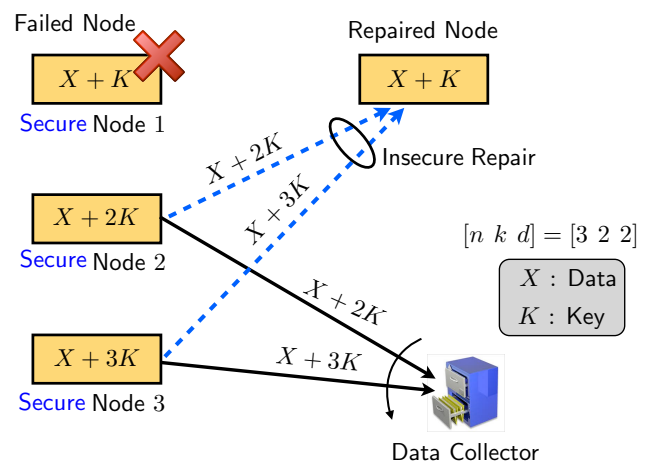

Fig. 1: (3, 2,2)-DSS with Type-I (node) Security.

abilities. On the other hand, information theoretic security offers provable security as it makes no assumption on the capabilities of the adversary. Security in a DSS against TypeII attacks was introduced in [9] where, linear codes that achieve the optimal tradeoff region in the bandwidth limited regime were proposed. In the context of DSS, secure exact repair regenerating codes are beneficial as the functional repair process may reveal additional information such as coding coefficients that are used to regenerate a functionally equivalent node [10]. Optimal exact repair codes that are secure against eavesdropping have been explored for the MSR and MBR points in [8], [11], [12]. The codes developed in [11] achieve the MBR point for all $(n, k, d)$ configurations with any $l<k$. The MSR code in [11] was shown to be optimal for Type-I attacks while it was shown to be optimal for Type-II attacks with $l=1$ in [8]. The maximum file size $\mathcal{B}$ that can be securely stored using linear MSR codes with exact repair was studied in [12] and proved to be optimal for $d=n-1$.

As an example, consider the $(3,2,2)$-DSS in the presence of a Type-I adversary that can only read the contents of any $l=1$ node. A secure exact repair code for this problem is shown in Fig. 1. When the 1 st node fails, the other nodes send their data contents using which (2 linear combinations of 2 symbols) it can recover its initial data contents (exact repair). Since the eavesdropper is unaware of the key $K$, it cannot recover the data symbol $X$ by wiretapping any single node in the DSS. Now, consider the same $(3,2,2)$-DSS in the presence of a more powerful Type-II adversary that has access to the repair data of any one node. It is clear that the scheme in Fig. 1 is not secure, as the wiretapper can use $(X+2 K, X+3 K)$ to recover $X$. Thus, a different secure exact repair DSS that can handle a Type-II adversary is shown in Fig. 2. It is seen that the storage capacity should be increased to $\alpha=2$ in this case when compared to the Type-I attack (where $\alpha=1$ ) as the eavesdropper can wiretap the repair process. Since the eavesdropper is not aware of the keys $K_{1}, K_{2}$, it cannot decode $X$ even if it observes the repair data of any single node.

From these schemes, it is seen that the secure repair scheme in Fig. 2 requires higher storage per-node $(\alpha=2)$ compared to the secure node scheme in Fig. $1(\alpha=1)$. We ask a basic question: is it possible to store a file of size $\mathcal{B}=1$, over nodes with $\alpha<2$ and with repair bandwidth $\beta=1$ while still preserving the security of the repair process? In this paper, we

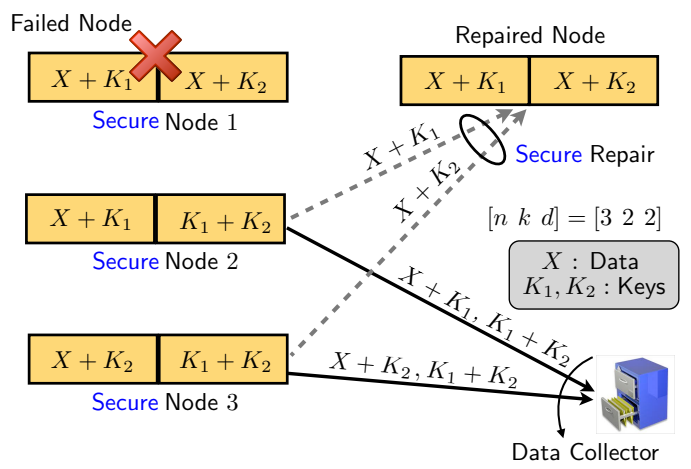

Fig. 2: (3, 2, 2)-DSS with Type-II (repair) Security.

argue that if secure and exact repair requirements are imposed, then the storage per-node cannot be smaller than $\alpha=2$ and hence the scheme in Fig. 2 is optimal.

In the sequel, we characterize the optimal exact repair region for the $(3,2,2)$-DSS with $l=1$ and the $(4,3,3)$ DSS with $l=1,2$ under Type-II attacks. Novel converse proofs that establish these optimal secure tradeoff regions are presented. Due to space limitations, we only present the converse proof for the $(3,2,2)$-DSS in the Appendix. For a complete characterization of the secure storage-vs-exact repair-bandwidth tradeoff regions under Type-I and Type-II attacks, please see the longer version of this paper [13].

\section{SYSTEM MODEL}

A $(n, k, d, \alpha, \beta, \mathcal{B})$ DSS consists of $n$ storage nodes that store a file $F$ of size $\mathcal{B}$ across $n$ nodes, with each node storing up to $\alpha$ units of data. A data collector connects to any $k<n$ nodes in order to reconstruct the file $F$. This is known as the MDS property of the DSS [3]. We focus on single node failures in which at any given point only one node in the system could fail. For the repair of a failed node, any $d$ out of the remaining $(n-1)$ alive nodes send $\beta \leq \alpha$ units of data in order to aid the repair process. The parameter $d \beta$ is referred to as the total repair bandwidth. From an information theoretic perspective, the goal is to store a file $F$, whose entropy is $\mathcal{B}$, i.e., $H(F)=\mathcal{B}$. Let $W_{i}$ denote the storage content at node $i$, for $i=1,2 \ldots, n$. Hence,

$$
H\left(W_{i}\right) \leq \alpha, \quad \forall i=1,2, \ldots, n .
$$

Due to the MDS property we also have

$$
H\left(F \mid W_{\{k\}}\right)=0,
$$

where $W_{\{k\}}$ is the data stored in any subset of $k$ storage nodes. Let $S_{i j}$ denote the data sent by node $i$ to repair node $j$. Due to the repair bandwidth constraint, we have

$$
H\left(S_{i j}\right) \leq \beta,
$$

and for exact repair of node $j$ from $d$ nodes, we also have

$$
H\left(W_{j} \mid S_{r_{1} j}, S_{r_{2} j}, \ldots, S_{r_{d} j}\right)=0,\left\{r_{i}\right\}_{i=1}^{d} \in[1, n] \neq j .
$$

Since $S_{i j}$ is a function of the data stored in node $i$, we have $H\left(S_{i j} \mid W_{i}\right)=0$. For the repair of any $l<k$ nodes to be 


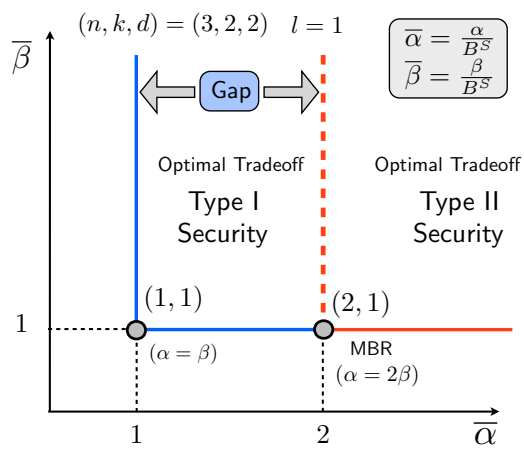

Fig. 3: Secure $(\alpha, \beta)$ tradeoff for $(3,2,2)$-DSS and $l=1$. secure, we require

$$
I\left(F ; S_{n_{1}}, S_{n_{2}}, \ldots, S_{n_{l}}\right)=0,
$$

where $S_{n_{i}}$ is the data (downloaded from $d$ repair nodes) used in the repair of node $n_{i}$.

The constraints regarding file-regeneration and exact repair with respect to a $(3,2,2)$ DSS are as follows:

File Regeneration:

$$
H\left(F \mid W_{1}, W_{2}\right)=H\left(F \mid W_{1}, W_{3}\right)=H\left(F \mid W_{2}, W_{3}\right)=0 .
$$

Exact Repair:

$H\left(W_{1} \mid S_{21}, S_{31}\right)=H\left(W_{2} \mid S_{12}, S_{32}\right)=H\left(W_{3} \mid S_{13}, S_{23}\right)=0$.

Repair data functionality:

$H\left(S_{12}, S_{13} \mid W_{1}\right)=H\left(S_{21}, S_{23} \mid W_{2}\right)=H\left(S_{31}, S_{32} \mid W_{1}\right)=0$.

For this example, $l$ can be either 0 or 1 . The non-trivial case is $l=1$, for which the Type-II security constraints are:

$$
I\left(F ; S_{21}, S_{31}\right)=I\left(F ; S_{12}, S_{32}\right)=I\left(F ; S_{13}, S_{23}\right)=0 .
$$

The secrecy capacity of a DSS under Type-II attacks is defined as the maximum file size that can be stored under the storage (2), file regeneration (3), repair bandwidth (4), exact repair (5) and Type-II secrecy constraints. Formally,

$$
\mathcal{B}_{I I}^{S}=\max _{(2)-(6)} H(F) \text {. }
$$

The study of distributed storage systems in the presence of a passive eavesdropper was initiated in [9]. It was shown that for any $(n, k, d)$-DSS with either Type-I or Type-II secrecy constraint (characterized by the parameter $l$ ), the following is an upper bound on the maximum secure file size $\mathcal{B}^{S}$ :

$$
\mathcal{B}^{S} \leq \sum_{i=l}^{k-1} \min (\alpha,(d-i) \beta) .
$$

Intuitively, this result can be interpreted as follows: in presence of a wiretapper, as $l$ nodes are compromised, at most $(k-l)$ nodes can help in recovering the entire file while keeping it secure from the eavesdropper. Hence the summation (compared to (1)) is over $(k-l)$ nodes as opposed to $k$ nodes [11]. We next show that there exists a significant gap between the optimal secure storage-vs-exact repair-bandwidth tradeoff region and the upper bound (8).

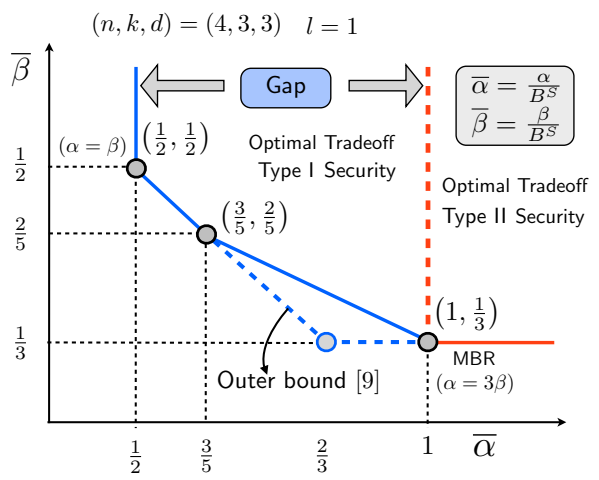

Fig. 4: Secure $(\alpha, \beta)$ tradeoff for $(4,3,3)$-DSS and $l=1$.

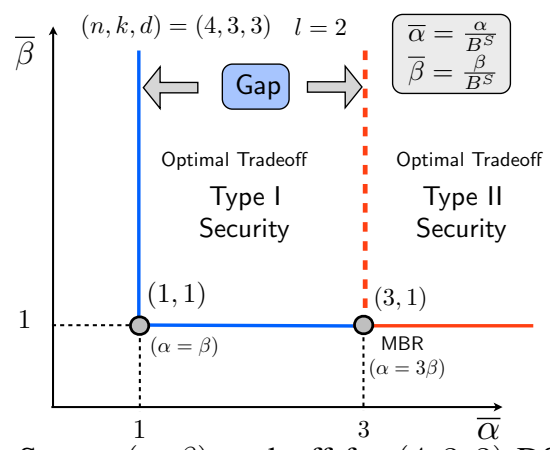

Fig. 5: Secure $(\alpha, \beta)$ tradeoff for $(4,3,3)$-DSS and $l=2$.

\section{Main Results}

In this section, we outline the main theorems that describe the optimal secure storage-vs-exact repair-bandwidth tradeoffs (in short referred to as $(\alpha, \beta)$-tradeoff region) under the exact repair and Type-II security constraints.

Theorem 1: The optimal $(\alpha, \beta)$-tradeoff region for the $(3,2,2)$-DSS with $l=1$, under exact repair and Type-II attack is given by:

$$
\mathcal{B}_{I I}^{S} \leq \min \left(\frac{\alpha}{2}, \beta\right) .
$$

The upper bound (8) in this case corresponds to $\mathcal{B}^{S} \leq$ $\min (\alpha, \beta)$ which indicates that there is a gap between this and the achievable tradeoff region (9). The main contribution of this paper is the information-theoretic converse proof for the bound (9) given in the Appendix.

Theorem 2: The optimal $(\alpha, \beta)$-tradeoff region for $(4,3,3)$ DSS with $l=1$, under exact repair and Type-II security constraints is given by:

$$
\mathcal{B}_{I I}^{S} \leq \min (\alpha, 3 \beta) .
$$

The upper bound (8) for the $(4,3,3)$ DSS with $l=1$, is given by $\mathcal{B}^{S} \leq \min (\alpha, \beta)+\min (\alpha, 2 \beta)$. This is different from the optimal region given by (10). This gap indicates that the maximum file size that can be securely stored is smaller than the file size obtained using (8).

Theorem 3: The optimal $(\alpha, \beta)$-tradeoff regions for $(4,3,3)$-DSS with $l=2$ under exact repair and Type-II attack is given by:

$$
\mathcal{B}_{I I}^{S} \leq \min \left(\frac{\alpha}{3}, \beta\right)
$$


This optimal tradeoff region is different from the region $\mathcal{B}^{S} \leq \min (\alpha, \beta)$ given by (8). Due to space limitations, the converse proofs for the $(4,3,3)$-DSS i.e., Theorems 2 and 3 are omitted. For these proofs, please refer to [13].

Figs. 3-5 show the optimal $(\alpha, \beta)$ tradeoff regions described by Theorems 1-3. From these theorems, it is seen that the only efficient point in the optimal $(\alpha, \beta)$-tradeoff region for the Type-II constraints is the MBR point where $\alpha=d \beta$. However, this is different from the optimal tradeoff-region achievable under Type-I constraints as seen in Figs. 3-5. Thus there is a gap between the optimal regions achievable under these two constraints i.e., the file size that can be securely stored under Type-II constraints is lower than the file size achieved under Type-I constraint [13]. The optimal $(\alpha, \beta)$-tradeoff regions under Type-I and Type-II constraints is completely characterized in [13].

\section{Achievability Proofs}

As mentioned earlier, the MBR point is the only achievable point in the presence of a Type-II adversary. The MBR point is defined by the $(\alpha, \beta)$ pair that satisfy $\alpha=d \beta$ [3]. Substituting this in (8), we get that the optimal secure file size $\mathcal{B}^{S}$ must satisfy

$$
\mathcal{B}^{S} \leq\left(k d-\left(\begin{array}{l}
k \\
2
\end{array}\right)\right) \beta-\left(l d-\left(\begin{array}{l}
l \\
2
\end{array}\right)\right) \beta
$$

Notice that (12) is identical to the tradeoff regions specified by Theorems $1-3$ for the $(3,2,2)$ and $(4,3,3)$ DSS with the corresponding values of $l$. This equivalence is further explained below.

Secure codes that achieve this MBR point for a general $(n, k, d)$ DSS with any $l<k$ compromised nodes have been described in [11] for the Type-II attacks. For example, in the $(3,2,2)$ DSS with $l=1$, the MBR point in (12) is simplified as $\mathcal{B}^{S}=\beta ; \alpha=2 \beta$, which is the $\alpha=2, \beta=1, \mathcal{B}^{S}=1$ point shown in Fig. 3, whose achievability is given in Fig. 2. In the $(4,3,3)$ DSS with $l=1,(12)$ is given by $\mathcal{B}^{S}=3 \beta ; \alpha=3 \beta$, which is also the $\alpha=3, \beta=1, \mathcal{B}^{S}=3$ point shown in Fig. 4. Along similar lines, with $l=2$, the MBR point in (12) is given by $\mathcal{B}^{S}=\beta ; \alpha=3 \beta$, i.e., the $\alpha=3, \beta=1, \mathcal{B}^{S}=1$ point shown in Fig. 5 (for more details, please see [11]).

Remark 1: Notice that the $(\alpha, \beta)=(3,1)$ pair (MBR point) is achievable for the $(4,3,3)$ DSS in the presence of a Type-II adversary, either when $l=1$ or $l=2$. However, the maximum file size that can be securely stored when $l=1$ is $\mathcal{B}^{S}=3$ while it is $\mathcal{B}^{S}=1$ when $l=2$. Intuitively, when 2 nodes in the DSS are compromised, the secrecy constraints are stringent compared to the case when only one node is compromised. Hence the file size that can be securely stored decreases when the number of compromised nodes increases.

\section{CONCLUSION}

Securing distributed storage systems against passive eavesdropping attacks is addressed in this paper. A complete characterization of the storage-bandwidth tradeoff region is provided for the $(3,2,2),(4,3,3)$ distributed storage systems under exact repair and Type-II secrecy constraints. Novel converse proofs that validate these optimal tradeoff regions are presented. As expected, the file size that can be securely stored decreases when the number of compromised nodes increases in the DSS. The gap in the file size that can be securely stored under Type-I and Type-II attacks increases as $n$ increases, thereby indicating the severe limitations of the DSS under Type-II attacks. A complete characterization of the optimal tradeoff region under Type-I and II adversaries is available in [13]. Extending these results to a general $(n, k, d)$ DSS is part of our ongoing work.

\section{REFERENCES}

[1] K. V. Rashmi, N. B. Shah, D. Gu, H. Kuang, D. Borthakur and K. Ramchandran, "A Solution to the Network Challenges of Data Recovery in Erasure-coded Distributed Storage Systems: A Study on the Facebook Warehouse Cluster", in arXiv:1309.0186, Sept. 2013.

[2] K. V. Rashmi, N. B. Shah and P. V. Kumar, "Enabling node repair in any erasure code for distributed storage", in arXiv:1101.0133, Jun. 2011.

[3] A. G. Dimakis, P. B. Godfrey, Y. Wu, M. Wainwright and K. Ramchandran, "Network coding for distributed storage systems," IEEE Trans. Inf. Theory, vol. 56, no. 9, pp. 4539-4551, Sept. 2010.

[4] R. Ahlswede, N. Cai, S.-Y. R. Li and R. W. Yeung, "Network information flow," IEEE Trans. Inf. Theory, vol. 46, pp. 1204-1216, Jul. 2000.

[5] N. B. Shah, K. V. Rashmi, P. V. Kumar and K. Ramchandran, "Distributed storage codes with repair-by-transfer and non-achievability of interior points on the storage-bandwidth tradeoff," IEEE Trans. Inf. Theory, vol. 58, no. 3, pp. 1837-1852, Mar. 2012.

[6] V. Cadambe, S. Jafar, H. Maleki, K. Ramchandran and C. Suh, "Asymptotic interference alignment for optimal repair of MDS codes in distributed storage," IEEE Trans Inf. Theory, vol. 59, no. 5, pp. 29742987, May. 2013.

[7] C. Tian, "Rate region of the $(4,3,3)$ exact-repair regenerating codes," in Proc. Intern. Symp. Inf Theory, ISIT, Istanbul, Turkey, Jun. 2013.

[8] A. S. Rawat, O. O. Koyluoglu, N. Silberstein, and S. Vishwanath, "Optimal locally repairable and secure codes for distributed storage systems," in arXiv:1210.6954, Aug. 2013.

[9] S. Pawar, S. El Rouayheb, and K. Ramchandran, "Securing dynamic distributed storage systems against eavesdropping and adversarial attacks," IEEE Trans. Inf. Theory, vol. 58, pp. 6734-6753, Mar. 2012.

[10] O. O. Koyluoglu, A. S. Rawat, and S. Vishwanath, "Secure Cooperative Regenerating Codes for Distributed Storage Systems,", in arXiv: 1210.3664 , Oct. 2012.

[11] N. B. Shah, K. V. Rashmi, and P. V. Kumar, "Information-theoretically secure regenerating codes for distributed storage," in Proc. IEEE Global Commun. Conf., GLOBECOM, Houston, TX, Dec. 2011.

[12] S. Goparaju, S. El Rouayheb, R. Calderbank and H. Vincent Poor, "Data Secrecy in Distributed Storage Systems under Exact Repair," in Proc. IEEE International Symposium on Network Coding, NETCOD, Calgary, Canada, Jun. 2013.

[13] R. Tandon, S. Amuru, T. C. Clancy and R. M. Buehrer, "Towards Optimal Secure Distributed Storage Systems with Exact Repair", in arXiv:1310.0054, Oct. 2013.

\section{APPENDIX}

\section{A. Proof of Theorem 1: $(3,2,2)$-DSS, $l=1$}

Note that from (8), we have the following existing upper bound for Type-II secrecy constraint:

$$
\mathcal{B}_{I I}^{S} \leq \min (\alpha, \beta) \text {. }
$$

In the remainder of this section, we will improve upon this bound for Type-II secrecy constraint and show that

$$
\mathcal{B}_{I I}^{S} \leq \min \left(\frac{\alpha}{2}, \beta\right)
$$

From (13), it is clear that $\mathcal{B}_{I I}^{S} \leq \beta$. Hence, it suffices to show that $\mathcal{B}_{I I}^{S} \leq \frac{\alpha}{2}$. We first recall the problem constraints. 
File regeneration from any $k=2$ nodes:

$$
H\left(F \mid W_{i}, W_{j}\right)=0, \forall i, j=1,2,3 \& i \neq j .
$$

Exact repair requirements:

$$
\begin{aligned}
& H\left(W_{1} \mid S_{21}, S_{31}\right)=0 \\
& H\left(W_{2} \mid S_{12}, S_{32}\right)=0 \\
& H\left(W_{3} \mid S_{13}, S_{23}\right)=0 .
\end{aligned}
$$

Secure repair of any $l=1$ node:

$$
\begin{aligned}
& I\left(F ; S_{21}, S_{31}\right)=0 \\
& I\left(F ; S_{12}, S_{32}\right)=0 \\
& I\left(F ; S_{13}, S_{23}\right)=0 .
\end{aligned}
$$

Repair data from a node is a function of stored data:

$$
\begin{aligned}
& H\left(S_{12}, S_{13} \mid W_{1}\right)=0 \\
& H\left(S_{21}, S_{23} \mid W_{2}\right)=0 \\
& H\left(S_{31}, S_{32} \mid W_{3}\right)=0 .
\end{aligned}
$$

From the above constraints, we note that the file $F$ can be reconstructed from 6 combinations of three repair random variables. These 6 cases are as follows:

$$
\begin{aligned}
& H\left(F \mid S_{21}, S_{31}, S_{23}\right)=0 \\
& H\left(F \mid S_{21}, S_{31}, S_{32}\right)=0 \\
& H\left(F \mid S_{12}, S_{32}, S_{13}\right)=0 \\
& H\left(F \mid S_{12}, S_{32}, S_{31}\right)=0 \\
& H\left(F \mid S_{13}, S_{23}, S_{12}\right)=0 \\
& H\left(F \mid S_{13}, S_{23}, S_{21}\right)=0 .
\end{aligned}
$$

As these conditions are symmetric in $1 \leftrightarrow 2 \leftrightarrow 3$, it suffices to prove any one of them. Hence, we prove (25) as follows:

$$
\begin{aligned}
H\left(F \mid S_{21}, S_{31}, S_{23}\right) & \stackrel{(16)}{=} H\left(F \mid S_{21}, S_{31}, W_{1}, S_{23}\right) \\
& \stackrel{(22)}{=} H\left(F \mid S_{21}, S_{31}, W_{1}, S_{13}, S_{23}\right) \\
& \stackrel{(18)}{=} H\left(F \mid S_{21}, S_{31}, W_{1}, S_{13}, S_{23}, W_{3}\right) \\
& \leq H\left(F \mid W_{1}, W_{3}\right) \stackrel{(15)}{=} 0 .
\end{aligned}
$$

From the constraint of secure repair of node 1 (Type-II secrecy constraint) implied by (19), we have the following set of inequalities:

$$
\begin{aligned}
& H(F) \stackrel{(19)}{=} H\left(F \mid S_{21}, S_{31}\right) \\
& =H\left(F, S_{21}, S_{31}\right)-H\left(S_{21}, S_{31}\right) \\
& =H\left(F, S_{21}, S_{31}\right)-H\left(S_{21}, S_{31}, W_{1}\right)+H\left(W_{1} \mid S_{21}, S_{31}\right) \\
& \stackrel{(16)}{=} H\left(F, S_{21}, S_{31}\right)-H\left(S_{21}, S_{31}, W_{1}\right) \\
& \stackrel{(22)}{=} H\left(F, S_{21}, S_{31}\right)-H\left(S_{21}, S_{31}, S_{12}, S_{13}, W_{1}\right) \\
& \leq H\left(F, S_{21}, S_{31}\right)-H\left(S_{21}, S_{31}, S_{12}, S_{13}\right) \\
& =H\left(F, S_{21}, S_{31}\right)-H\left(S_{12}, S_{21}\right) \\
& -H\left(S_{31}, S_{13} \mid S_{12}, S_{21}\right) \text {. }
\end{aligned}
$$

Similarly, from the constraint of secure repair of node 2 implied by (20), we have the following set of inequalities:

$$
\begin{aligned}
& H(F) \stackrel{(20)}{=} H\left(F \mid S_{12}, S_{32}\right) \triangleq H\left(F, S_{12}, S_{32}\right)-H\left(S_{12}, S_{32}\right) \\
& =H\left(F, S_{12}, S_{32}\right)-H\left(S_{12}, S_{32}, W_{2}\right)+H\left(W_{2} \mid S_{12}, S_{32}\right) \\
& \stackrel{(17)}{=} H\left(F, S_{12}, S_{32}\right)-H\left(S_{12}, S_{32}, W_{2}\right) \\
& \stackrel{(23)}{=} H\left(F, S_{12}, S_{32}\right)-H\left(S_{12}, S_{32}, S_{21}, S_{23}, W_{2}\right) \\
& \leq H\left(F, S_{12}, S_{32}\right)-H\left(S_{12}, S_{32}, S_{21}, S_{23}\right) \\
& =H\left(F, S_{12}, S_{32}\right)-H\left(S_{12}, S_{21}\right) \\
& -H\left(S_{32}, S_{23} \mid S_{12}, S_{21}\right) \text {. }
\end{aligned}
$$

Summing up (32) and (33), we get

$$
\begin{aligned}
2 H(F) \leq & H\left(F, S_{21}, S_{31}\right)+H\left(F, S_{12}, S_{32}\right) \\
& -2 H\left(S_{12}, S_{21}\right)-H\left(S_{31}, S_{13} \mid S_{12}, S_{21}\right) \\
& -H\left(S_{32}, S_{23} \mid S_{12}, S_{21}\right) \\
\leq & H\left(F, S_{21}, S_{31}\right)+H\left(F, S_{12}, S_{32}\right) \\
& -2 H\left(S_{12}, S_{21}\right)-H\left(S_{31}, S_{13}, S_{32}, S_{23} \mid S_{12}, S_{21}\right) \\
= & H\left(F, S_{21}, S_{31}\right)+H\left(F, S_{12}, S_{32}\right) \\
& -H\left(S_{12}, S_{21}\right)-H\left(S_{12}, S_{21}, S_{31}, S_{13}, S_{32}, S_{23}\right) \\
\leq & H\left(F, S_{21}, S_{31}\right)+H\left(F, S_{12}, S_{32}\right) \\
& -H\left(S_{12}, S_{21}\right)-H\left(S_{12}, S_{32}, S_{13}\right) \\
= & H\left(F, S_{21}, S_{31}\right)+H\left(F, S_{12}, S_{32}\right) \\
& -H\left(S_{12}, S_{21}\right)-H\left(S_{12}, S_{32}, S_{13}, F\right) \\
& +H\left(F \mid S_{12}, S_{32}, S_{13}\right) \\
(27) & H\left(F, S_{21}, S_{31}\right)+H\left(F, S_{12}, S_{32}\right) \\
& -H\left(S_{12}, S_{21}\right)-H\left(S_{12}, S_{32}, S_{13}, F\right) \\
\leq & H\left(F, S_{21}, S_{31}\right)+H\left(F, S_{12}, S_{32}\right) \\
& -H\left(S_{12}, S_{21}\right)-H\left(S_{12}, S_{32}, F\right) \\
= & H\left(F, S_{21}, S_{31}\right)-H\left(S_{12}, S_{21}\right) .
\end{aligned}
$$

We next consider the first term in (34) and bound it as follows:

$$
\begin{aligned}
H\left(F, S_{21}, S_{31}\right) & \leq H\left(F, W_{3}, S_{21}, S_{31}\right) \\
& =H\left(W_{3}, S_{21}, S_{31}\right)+H\left(F \mid W_{3}, S_{21}, S_{31}\right) \\
& \stackrel{(16)}{=} H\left(W_{3}, S_{21}, S_{31}\right)+H\left(F \mid W_{3}, W_{1}, S_{21}, S_{31}\right) \\
& \stackrel{(15)}{=} H\left(W_{3}, S_{21}, S_{31}\right) \\
& \stackrel{(24)}{=} H\left(W_{3}, S_{21}\right) \\
& \leq H\left(W_{3}, S_{21}, S_{12}\right) \\
& \leq H\left(W_{3}\right)+H\left(S_{21}, S_{12}\right) \\
& \leq \alpha+H\left(S_{21}, S_{12}\right) .
\end{aligned}
$$

Substituting (35) in (34), we arrive at

$$
\begin{aligned}
2 H(F) & \leq \alpha+H\left(S_{21}, S_{12}\right)-H\left(S_{12}, S_{21}\right) \\
& =\alpha,
\end{aligned}
$$

which implies that $H(F) \leq \frac{\alpha}{2}$ and hence $\mathcal{B}_{I I}^{S} \leq \frac{\alpha}{2}$. 